\title{
Promising Zintl-Phase Thermoelectric Compound SrAgSb
}

Weiming Zhang ${ }^{\text {a\# }}$, Chen Chen ${ }^{\text {a\# }}$, Honghao Yao ${ }^{\mathrm{a}}$, Wenhua Xue ${ }^{\mathrm{b}}$, Shan $\mathrm{Li}^{\mathrm{a}}$, Fengxian Bai ${ }^{\mathrm{a}}$, Yifang Huang ${ }^{\mathrm{a}}$, Xiaofang $\mathrm{Li}^{\mathrm{a}}$, Xi Lin ${ }^{\mathrm{a}}$, Feng Cao ${ }^{\mathrm{c}}$, Jiehe Suid, Shufang Wang ${ }^{\mathrm{e}}$, Bo Yư ${ }^{\mathrm{f}}$, Yumei Wang ${ }^{\mathrm{b}}$, Xingjun Liu ${ }^{\text {a, d }}$, Qian Zhang, ${ }^{\text {a }}{ }^{*}$

${ }^{a}$ School of Materials Science and Engineering, and Institute of Materials Genome \& Big Data, Harbin Institute of Technology, Shenzhen, Guangdong 518055, P.R. China

${ }^{b}$ Beijing National Laboratory for Condensed Matter Physics, Institute of Physics, Chinese Academy of Science, Beijing 100190, P.R. China

${ }^{c}$ School of Science, Harbin Institute of Technology, Shenzhen, Guangdong 518055, P. R. China

${ }^{d}$ State Key Laboratory of Advanced Welding and Joining, Harbin Institute of Technology, Harbin, Heilongjiang 150001, P.R. China

${ }^{e}$ Hebei Key Laboratory of Optic-Electronic Information and Materials, College of Physics Science and Technology, Hebei University, Baoding 071002, China

${ }^{f}$ Ningbo Fengcheng Advanced Energy Materials Research Institute, Fenghua District, Ningbo, Zhejiang, 315500, China

*Corresponding author. Email: zhangqf@hit.edu.cn

\section{Lorenz number calculation:}

The Lorenz number is calculated based on the single parabolic band (SPB) model ${ }^{1,2}$. The detailed formulas are shown as follows:

$$
\begin{gathered}
L=\left(\frac{k_{\mathrm{B}}}{e}\right)^{2}\left\{\frac{(r+7 / 2) F_{r+5 / 2}\left(\eta_{\mathrm{F}}\right)}{(r+3 / 2) F_{r+1 / 2}\left(\eta_{\mathrm{F}}\right)}-\left[\frac{(r+5 / 2) F_{r+3 / 2}\left(\eta_{\mathrm{F}}\right)}{(r+3 / 2) F_{r+1 / 2}\left(\eta_{\mathrm{F}}\right)}\right]^{2}\right\} \\
S= \pm \frac{k_{\mathrm{B}}}{e}\left[\frac{(r+5 / 2) F_{r+3 / 2}\left(\eta_{\mathrm{F}}\right)}{(r+3 / 2) F_{r+1 / 2}\left(\eta_{\mathrm{F}}\right)}-\eta_{\mathrm{F}}\right] \\
F_{i}\left(\eta_{\mathrm{F}}\right)=\int_{0}^{\infty} \frac{x^{i} d x}{1+\exp \left(x-\eta_{\mathrm{F}}\right)} \\
\eta_{\mathrm{F}}=E_{\mathrm{F}} / k_{\mathrm{B}} T
\end{gathered}
$$

where $k_{\mathrm{B}}$ is the Boltzmann constant, $e$ is the electron charge, $\eta_{\mathrm{F}}$ is the reduced Fermi energy, $r$ is the scattering factor (here, acoustic phonon scattering is considered, $r=-1 / 2), F_{i}\left(\eta_{\mathrm{F}}\right)$ is the Fermi-Dirac integral.

\section{Reference}


1. Mao, J.; Kim, H. S.; Shuai, J.; Liu, Z.; He, R.; Saparamadu, U.; Tian, F.; Liu, W.; Ren, Z., Thermoelectric properties of materials near the band crossing line in $\mathrm{Mg}_{2} \mathrm{Sn}-\mathrm{Mg}_{2} \mathrm{Ge}-\mathrm{Mg}_{2} \mathrm{Si}$ system. Acta Mater. 2016, 103, 633-642.

2. Li, W.; Zhou, B.; Li, J., Single parabolic band behavior of thermoelectric p-type $\mathrm{Cu}_{4} \mathrm{Mn}_{2} \mathrm{Te}_{4} . J$. Alloys Compd. 2018, 753, 93-99.

2. Measurement of annealed $\mathrm{Sr}_{1.01} \mathrm{AgSb}$
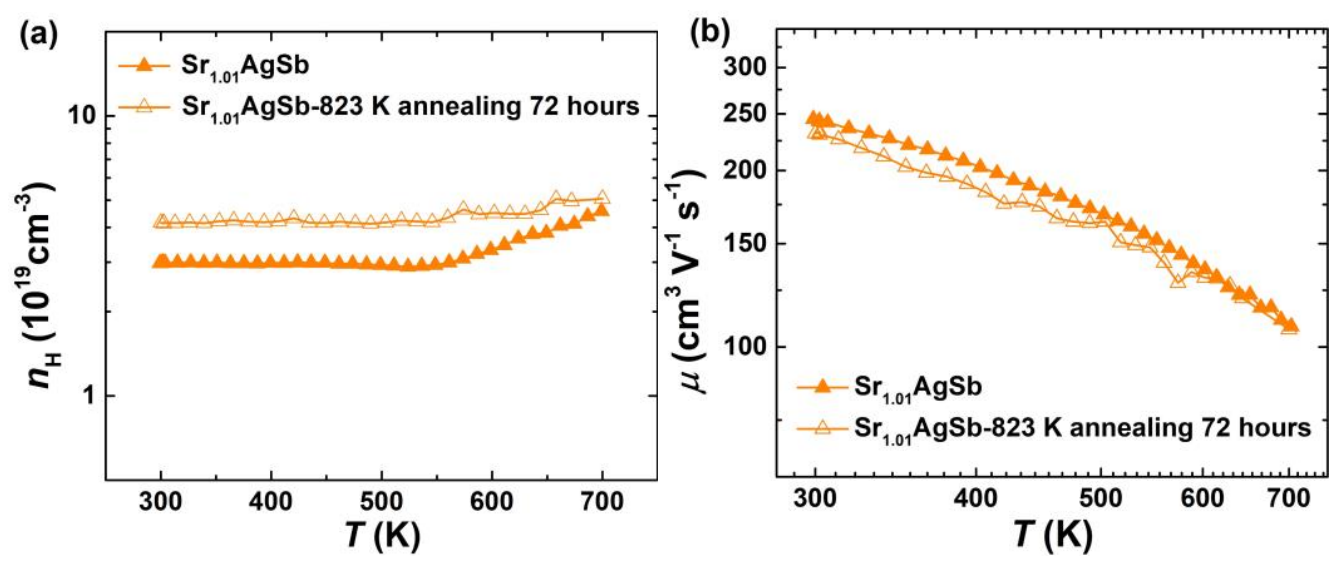

Figure S1. Hall carrier concentration and mobility of $\mathrm{Sr}_{1.01} \mathrm{AgSb}$ before and after annealing. 

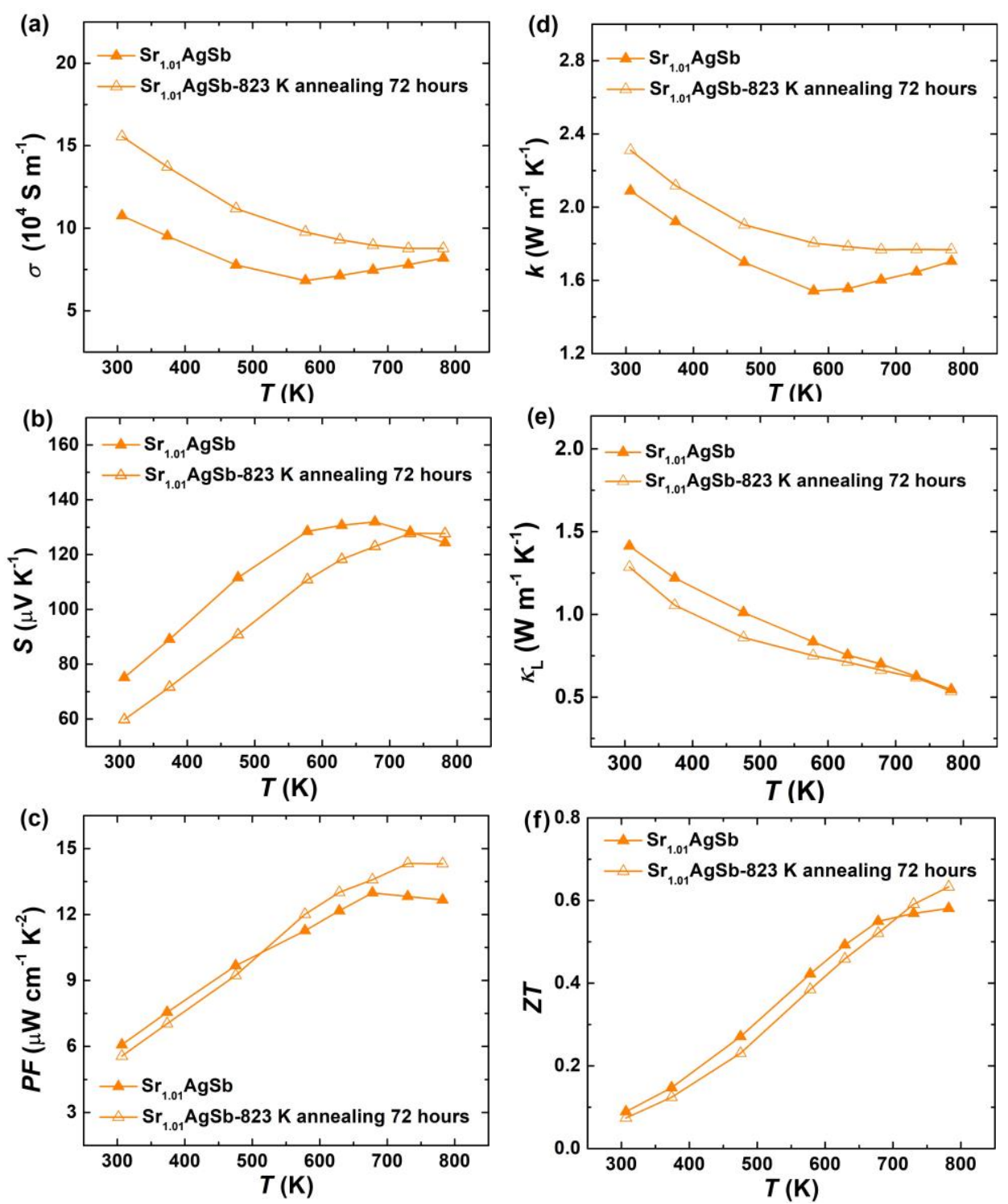

Figure S2. Temperature-dependent thermoelectric properties of $\mathrm{Sr}_{1.01} \mathrm{AgSb}$ before and after annealing. (a) Electrical conductivity, (b) Seebeck coefficient, (c) Power factor, (d) Thermal conductivity, (e) Lattice thermal conductivity, and (f) ZT.

\section{Specific heat of $\mathrm{Sr}_{x} \mathrm{AgSb}$}

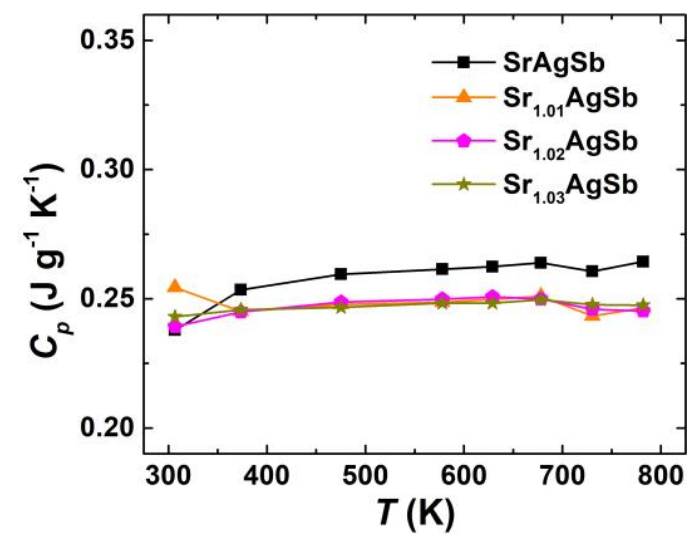

Figure S3. Temperature-dependent specific heat of $\operatorname{Sr}_{x} \mathrm{AgSb}(x=1,1.01,1.02$, and 1.03). 